\title{
INITIAL-BOUNDARY VALUE PROBLEMS FOR IMPULSIVE PARABOLIC FUNCTIONAL DIFFERENTIAL EQUATIONS
}

Abstract. Theorems on differential inequalities generated by an initialboundary value problem for impulsive parabolic functional differential equations are considered. Comparison results implying uniqueness criteria are proved.

1. Introduction. The theory of impulsive ordinary differential equations made its start in [10] and it was an object of many investigations in the last three decades ([3], [4]). This theory is richer than the corresponding theory without impulses due to some new features and phenomena such as: "beating", "merging", "dying" of solutions, loss of autonomy, etc.

In the recent years the theory of impulsive partial differential equations began to emerge ([1], [5], [7], [8]). It gives greater possibilities for mathematical simulation of evolutional processes in theoretical physics, chemistry, population dynamics, biotechnology, etc., which are characterized by the fact that the system parameters are subject to short term perturbations in time. The authors believe that this new theory will undergo a rapid development in the coming years.

In the present paper impulsive parabolic functional differential inequalities are considered. It is shown that the impulsive ordinary functional differential inequalities find application in the proofs of theorems concerning the estimates of solutions and in the uniqueness theory for impulsive parabolic functional differential equations.

1991 Mathematics Subject Classification: Primary 35R10; Secondary 35R45.

Key words and phrases: impulsive parabolic equations, initial-boundary value problems. 
We note that parabolic differential and functional differential inequalities with impulses are investigated in [2], [5], [6], [8].

2. Preliminaries. Let $E=[0, a) \times(-b, b), a>0, b=\left(b_{1}, \ldots, b_{n}\right) \in \mathbb{R}_{+}^{n}$, $\mathbb{R}_{+}=[0, \infty)$ and $B=\left[-\tau_{0}, 0\right] \times[-\tau, \tau]$, where $\tau_{0} \in \mathbb{R}_{+}, \tau=\left(\tau_{1}, \ldots, \tau_{n}\right)$ $\in \mathbb{R}_{+}^{n}$. We define $c=b+\tau, E_{0}=\left[-\tau_{0}, 0\right] \times[-c, c], \partial_{0} E=[0, a) \times([-c, c] \backslash$ $(-b, b)), E^{*}=E \cup E_{0} \cup \partial_{0} E$. For $\tau_{0}>0$ we put $B^{(-)}=\left[-\tau_{0}, 0\right) \times[-\tau, \tau]$.

Suppose that $0<x_{1}<\ldots<x_{k}<a$ are given numbers. We define

$$
\begin{aligned}
J_{0}=\left[-\tau_{0}, 0\right], \quad J=[0, a), \quad J_{\mathrm{imp}}=\left\{x_{1}, \ldots, x_{k}\right\}, \\
E_{\mathrm{imp}}=\left\{(x, y) \in E: x \in J_{\mathrm{imp}}\right\}, \\
\partial_{0} E_{\mathrm{imp}}=\left\{(x, y) \in \partial_{0} E: x \in J_{\mathrm{imp}}\right\}, \\
E_{\mathrm{imp}}^{*}=\left\{(x, y) \in E^{*}: x \in J_{\mathrm{imp}}\right\} .
\end{aligned}
$$

Let $C_{\mathrm{imp}}\left[E^{*}, \mathbb{R}\right]$ be the class of all functions $z: E^{*} \rightarrow \mathbb{R}$ such that:

(i) the restriction of $z$ to $E^{*} \backslash E_{\mathrm{imp}}^{*}$ is continuous,

(ii) for each $(x, y) \in E_{\mathrm{imp}}$, the limits

$$
\begin{aligned}
\lim _{\substack{t, s) \rightarrow(x, y) \\
t<x}} z(t, s) & =z\left(x^{-}, y\right), \\
\lim _{\substack{(t, s) \rightarrow(x, y) \\
t>x}} z(t, s) & =z\left(x^{+}, y\right)
\end{aligned}
$$

exist and $z(x, y)=z\left(x^{+}, y\right)$ for $(x, y) \in E_{\text {imp }}$.

In the same way we define the set $C_{\text {imp }}\left[\partial_{0} E, \mathbb{R}\right]$. If $z \in C_{\mathrm{imp}}\left[E^{*}, \mathbb{R}\right]$ and $(x, y) \in E_{\text {imp }}$ then we write $\Delta z(x, y)=z(x, y)-z\left(x^{-}, y\right)$.

Suppose that $z: E^{*} \rightarrow \mathbb{R}$ and $(x, y)=\left(x, y_{1}, \ldots, y_{n}\right) \in \bar{E}$, the closure of $E$. We define a function $z_{(x, y)}: B \rightarrow \mathbb{R}$ as follows:

$$
z_{(x, y)}(t, s)=z(x+t, y+s), \quad(t, s) \in B .
$$

Suppose that $\tau_{0}>0$. For the above $z$ and $(x, y)$ we also define $z_{\left(x^{-}, y\right)}$ : $B^{(-)} \rightarrow \mathbb{R}$ by

$$
z_{\left(x^{-}, y\right)}(t, s)=z(x+t, y+s), \quad(t, s) \in B^{(-)} .
$$

Assume that we have a sequence $\left\{t_{1}, \ldots, t_{r}\right\}$ such that $-\tau_{0} \leq t_{1}<\ldots<$ $t_{r} \leq 0$. Let $\Gamma_{i}=\left(t_{i}, t_{i+1}\right) \times[-\tau, \tau], i=1, \ldots, r-1$ and

$$
\begin{aligned}
& \Gamma_{0}= \begin{cases}\emptyset & \text { if }-\tau_{0}=t_{1}, \\
\left(-\tau_{0}, t_{1}\right) \times[-\tau, \tau] & \text { if }-\tau_{0}<t_{1},\end{cases} \\
& \Gamma_{r}= \begin{cases}\emptyset & \text { if } t_{r}=0, \\
\left(t_{r}, 0\right) \times[-\tau, \tau] & \text { if } t_{r}<0 .\end{cases}
\end{aligned}
$$


Let $t_{0}=-\tau_{0}$ if $t_{1}>-\tau_{0}$ and $t_{r+1}=0$ if $t_{r}<0$. We denote by $C_{\mathrm{imp}}^{*}[B, \mathbb{R}]$ the class of all functions $w: B \rightarrow \mathbb{R}$ such that there exists a sequence $\left\{t_{1}, \ldots, t_{r}\right\}$ ( $r$ and $t_{1}, \ldots, t_{r}$ depend on $w$ ) satisfying:

(i) the functions $w_{\mid \Gamma_{i}}, i=0,1, \ldots, r$, are continuous,

(ii) for each $i, i=1, \ldots, r+1$ with $\left(t_{i}, s\right) \in B, t_{i}>-\tau_{0}$, the limit

$$
\lim _{\substack{(t, y) \rightarrow\left(t_{i}, s\right) \\ t<t_{i}}} w(t, y)=w\left(t_{i}^{-}, s\right)
$$

exists,

(iii) for each $i, i=0,1, \ldots, r$ with $\left(t_{i}, s\right) \in B, t_{i}<0$, the limit

$$
\lim _{\substack{(t, y) \rightarrow\left(t_{i}, s\right) \\ t>t_{i}}} w(t, y)=w\left(t_{i}^{+}, s\right)
$$

exists,

(iv) for each $\left(t_{i}, s\right) \in B, i=0,1, \ldots, r-1$, and for $i=r$ if $t_{r}<0$, we have $w\left(t_{i}, s\right)=w\left(t_{i}^{+}, s\right)$.

Let $C_{\mathrm{imp}}^{*}\left[B^{(-)}, \mathbb{R}\right]=\left\{w_{\mid B^{(-)}}: w \in C_{\mathrm{imp}}^{*}[B, \mathbb{R}]\right\}$ in the case $\tau_{0}>0$. Elements of the sets $C_{\mathrm{imp}}^{*}[B, \mathbb{R}]$ and $C_{\mathrm{imp}}^{*}\left[B^{(-)}, \mathbb{R}\right]$ will be denoted by the same symbols. It is easy to see that if $z \in C_{\text {imp }}\left[E^{*}, \mathbb{R}\right]$ and $(x, y) \in \bar{E}$, then $z_{(x, y)} \in C_{\mathrm{imp}}^{*}[B, \mathbb{R}]$ and $z_{\left(x^{-}, y\right)} \in C_{\mathrm{imp}}^{*}\left[B^{(-)}, \mathbb{R}\right]$ in the case $\tau_{0}>0$.

For $w \in C_{\mathrm{imp}}^{*}[B, \mathbb{R}]$ we define $\|w\|_{B}=\sup \{|w(t, s)|:(t, s) \in B\}$. We denote by $\|\cdot\|_{B^{(-)}}$the supremum norm in the space $C_{\mathrm{imp}}^{*}\left[B^{(-)}, \mathbb{R}\right]$.

Let $M[n]$ be the class of all matrices $\gamma=\left[\gamma_{i j}\right]_{1 \leq i, j \leq n}$, where $\gamma_{i j} \in \mathbb{R}$ and $\gamma_{i j}=\gamma_{j i}$.

Suppose that

$$
\begin{aligned}
\Omega & =\left(E \backslash E_{\mathrm{imp}}\right) \times \mathbb{R} \times C_{\mathrm{imp}}^{*}[B, \mathbb{R}] \times \mathbb{R}^{n} \times M[n], \\
\Omega_{\mathrm{imp}} & =\left(E_{\mathrm{imp}} \cup \partial_{0} E_{\mathrm{imp}}\right) \times \mathbb{R} \times C_{\mathrm{imp}}^{*}\left[B^{(-)}, \mathbb{R}\right]
\end{aligned}
$$

and $f: \Omega \rightarrow \mathbb{R}, g: \Omega_{\text {imp }} \rightarrow \mathbb{R}$ and $\varphi: E_{0} \cup \partial_{0} E \rightarrow \mathbb{R}$, where $\varphi_{\mid \partial_{0} E} \in$ $C_{\mathrm{imp}}\left[\partial_{0} E, \mathbb{R}\right]$, are given functions.

A function $z \in C_{\text {imp }}\left[E^{*}, \mathbb{R}\right]$ will be called a function of class $C_{\mathrm{imp}}^{(1,2)}\left[E^{*}, \mathbb{R}\right]$ if $z$ has continuous derivatives $D_{x} z(x, y), D_{y} z(x, y)$ and $D_{y y} z(x, y)$ for $(x, y)$ $\in E \backslash E_{\mathrm{imp}}$, where

$$
D_{y} z=\left(D_{y_{1}} z, \ldots, D_{y_{n}} z\right), \quad D_{y y} z=\left[D_{y_{i} y_{j}} z\right]_{1 \leq i, j \leq n} .
$$

A function $f: \Omega \rightarrow \mathbb{R}$ is said to be parabolic with respect to $z \in$ $C_{\mathrm{imp}}^{(1,2)}\left[E^{*}, \mathbb{R}\right]$ in $E \backslash E_{\mathrm{imp}}$ if for $(x, y) \in E \backslash E_{\mathrm{imp}}$ and for any $\gamma, s \in M[n]$ such that

$$
\sum_{i, j=1}^{n}\left(\gamma_{i j}-s_{i j}\right) \lambda_{i} \lambda_{j} \leq 0, \quad \lambda=\left(\lambda_{1}, \ldots, \lambda_{n}\right) \in \mathbb{R}^{n}
$$


we have

$$
f\left(x, y, z(x, y), z_{(x, y)}, D_{y} z(x, y), \gamma\right) \leq f\left(x, y, z(x, y), z_{(x, y)}, D_{y} z(x, y), s\right) .
$$

We consider the initial-boundary value problem:

(3) $D_{x} z(x, y)=f\left(x, y, z(x, y), z_{(x, y)}, D_{y} z(x, y), D_{y y} z(x, y)\right)$,

$$
(x, y) \in E \backslash E_{\mathrm{imp}},
$$

(4) $z(x, y)=\varphi(x, y), \quad(x, y) \in E_{0} \cup \partial_{0} E$,

(5) $\Delta z(x, y)=g\left(x, y, z\left(x^{-}, y\right), z_{\left(x^{-}, y\right)}\right), \quad(x, y) \in E_{\mathrm{imp}} \cup \partial_{0} E_{\mathrm{imp}}$.

For $f: \Omega \rightarrow \mathbb{R}, g: \Omega_{\mathrm{imp}} \rightarrow \mathbb{R}$ and $z \in C_{\mathrm{imp}}^{(1,2)}\left[E^{*}, \mathbb{R}\right]$ we write

$$
\begin{array}{r}
F[z](x, y)=D_{x} z(x, y)-f\left(x, y, z(x, y), z_{(x, y)}, D_{y} z(x, y), D_{y y} z(x, y)\right), \\
(x, y) \in E \backslash E_{\mathrm{imp}},
\end{array}
$$

and

$$
G[z](x, y)=\Delta z(x, y)-g\left(x, y, z\left(x^{-}, y\right), z_{\left(x^{-}, y\right)}\right), \quad(x, y) \in E_{\text {imp }} .
$$

\section{Main results}

3.1. Impulsive parabolic functional differential inequalities. We introduce

Assumption H1. Suppose that:

1. the function $f: \Omega \rightarrow \mathbb{R}$ of the variables $(x, y, p, w, q, s)$ is nondecreasing with respect to the functional argument $w$,

2. the function $g: \Omega_{\mathrm{imp}} \rightarrow \mathbb{R}$ of $(x, y, p, w)$ is non-decreasing with respect to $w$ and for each $(x, y) \in E_{\mathrm{imp}}$ and $w \in C_{\mathrm{imp}}^{*}\left[B^{(-)}, \mathbb{R}\right]$ the function $\delta(p)=p+g(x, y, p, w), p \in \mathbb{R}$, is non-decreasing on $\mathbb{R}$.

THEOREM 1. Suppose that:

1. Assumption $\mathrm{H} 1$ holds,

2. $u, v \in C_{\mathrm{imp}}^{(1,2)}\left[E^{*}, \mathbb{R}\right]$ satisfy the initial-boundary inequality

$$
u(x, y)<v(x, y), \quad(x, y) \in E_{0} \cup \partial_{0} E,
$$

3. the functional differential inequality

$$
F[u](x, y)<F[v](x, y), \quad(x, y) \in E \backslash E_{\mathrm{imp}},
$$

and the inequality for impulses

$$
G[u](x, y)<G[v](x, y), \quad(x, y) \in E_{\text {imp }},
$$

are satisfied,

4. $f$ is parabolic with respect to $u$ in $E \backslash E_{\mathrm{imp}}$.

Then

$$
u(x, y)<v(x, y) \quad \text { on } E^{*} .
$$


Proof. If (9) is false then the set $Z=\{x \in[0, a)$ : there exists $y \in$ $(-b, b)$ such that $u(x, y) \geq v(x, y)\}$ is non-empty. Defining $\widetilde{x}=\inf Z$ it follows from (6) that $\widetilde{x}>0$ and there exists $\widetilde{y} \in(-b, b)$ such that

$$
\begin{aligned}
& u(x, y)<v(x, y), \quad(x, y) \in E^{*} \cap\left(\left[-\tau_{0}, \widetilde{x}\right) \times \mathbb{R}^{n}\right), \\
& u(\widetilde{x}, \widetilde{y})=v(\widetilde{x}, \widetilde{y}) .
\end{aligned}
$$

There are two cases to be distinguished:

Case 1: $(\widetilde{x}, \widetilde{y}) \in E \backslash E_{\text {imp. }}$ Then

$$
D_{x}(u-v)(\widetilde{x}, \widetilde{y}) \geq 0, \quad D_{y}(u-v)(\widetilde{x}, \widetilde{y})=0
$$

and

$$
\sum_{i, j=1}^{n} D_{y_{i} y_{j}}(u-v)(\widetilde{x}, \widetilde{y}) \lambda_{i} \lambda_{j} \leq 0,
$$

for $\lambda=\left(\lambda_{1}, \ldots, \lambda_{n}\right) \in \mathbb{R}^{n}$, which leads to a contradiction with (7).

Case $2:(\widetilde{x}, \widetilde{y}) \in E_{\text {imp }}$. Then there exists $i, 1 \leq i \leq k$, such that $\widetilde{x}=x_{i}$. From (10) we have

$$
u\left(\widetilde{x}^{-}, \widetilde{y}\right) \leq v\left(\widetilde{x}^{-}, \widetilde{y}\right) .
$$

It follows from (8) and (11) that

$$
\begin{aligned}
u(\widetilde{x}, \widetilde{y})-v(\widetilde{x}, \widetilde{y})< & u\left(\widetilde{x}^{-}, \widetilde{y}\right)+g\left(\widetilde{x}, \widetilde{y}, u\left(\widetilde{x}^{-}, \widetilde{y}\right), u_{\left(\tilde{x}^{-}, \tilde{y}\right)}\right) \\
& -v\left(\widetilde{x}^{-}, \widetilde{y}\right)-g\left(\widetilde{x}, \widetilde{y}, v\left(\widetilde{x}^{-}, \widetilde{y}\right), v_{\left(\tilde{x}^{-}, \tilde{y}\right)}\right) \leq 0,
\end{aligned}
$$

which contradicts (10).

Hence $Z$ is empty and the statement (9) follows.

Remark 1 . In Theorem 1 we can assume instead of (7), (8) that

$$
\begin{array}{ll}
F[u](x, y)<F[v](x, y) & \text { for }(x, y) \in T \backslash E_{\mathrm{imp}}, \\
G[u](x, y)<G[v](x, y) & \text { for }(x, y) \in T \cap E_{\mathrm{imp}},
\end{array}
$$

where

$T=\{(x, y) \in E: u(t, s)<v(t, s)$ for $(t, s) \in E, t \in[0, x), u(x, y)=v(x, y)\}$.

Now we consider weak impulsive parabolic functional differential inequalities.

Assumption H2. Suppose that:

1. $\sigma:\left([0, a] \backslash J_{\mathrm{imp}}\right) \times \mathbb{R}_{+} \rightarrow \mathbb{R}_{+}$is continuous and $\sigma(x, 0)=0$ for $x \in[0, a] \backslash J_{\mathrm{imp}}$, 
2. $\sigma_{0}: J_{\mathrm{imp}} \times \mathbb{R}_{+} \rightarrow \mathbb{R}_{+}$is continuous, $\sigma_{0}(x, 0)=0$ for $x \in J_{\mathrm{imp}}$ and the right-hand maximal solution of the problem

$$
\begin{aligned}
& \alpha^{\prime}(x)=\sigma(x, \alpha(x)), \quad x \in J \backslash J_{\mathrm{imp}}, \\
& \alpha(0)=0, \\
& \Delta \alpha(x)=\sigma_{0}\left(x, \alpha\left(x^{-}\right)\right), \quad x \in J_{\mathrm{imp}},
\end{aligned}
$$

is $\alpha(x)=0, x \in J$,

3. $f: \Omega \rightarrow \mathbb{R}$ satisfies the inequality

$f(x, y, p, w, q, s)-f(x, y, \bar{p}, \bar{w}, q, s)$

$$
\geq-\sigma\left(x, \max \left\{\bar{p}-p,\|\bar{w}-w\|_{B}\right\}\right) \quad \text { on } \Omega,
$$

where $p \leq \bar{p}$ and $w \leq \bar{w}$,

4. for $(x, y, p, w) \in E_{\mathrm{imp}} \times \mathbb{R} \times C_{\mathrm{imp}}^{*}\left[B^{(-)}, \mathbb{R}\right]$ we have

$$
g(x, y, p, w)-g(x, y, \bar{p}, \bar{w}) \geq-\sigma_{0}\left(x, \max \left\{\bar{p}-p,\|\bar{w}-w\|_{B^{(-)}}\right\}\right),
$$

where $p \leq \bar{p}, w \leq \bar{w}$.

THEOREM 2. Suppose that:

1. Assumptions $\mathrm{H} 1$ and $\mathrm{H} 2$ hold,

2. $u, v \in C_{\mathrm{imp}}^{(1,2)}\left[E^{*}, \mathbb{R}\right]$ and

$$
u(x, y) \leq v(x, y) \quad \text { on } E_{0} \cup \partial_{0} E,
$$

3. the functional differential inequality

$$
F[u](x, y) \leq F[v](x, y), \quad(x, y) \in E \backslash E_{\mathrm{imp}},
$$

and the inequality for impulses

$$
G[u](x, y) \leq G[v](x, y), \quad(x, y) \in E_{\mathrm{imp}},
$$

are satisfied,

4. $f$ is parabolic with respect to $u$ in $E \backslash E_{\mathrm{imp}}$.

Then $u(x, y) \leq v(x, y)$ on $E^{*}$.

Proof. Suppose that $a_{0} \in\left(x_{k}, a\right)$. We prove that

$$
u(x, y) \leq v(x, y)
$$

for $(x, y) \in\left(\left[-\tau_{0}, a_{0}\right) \times \mathbb{R}^{n}\right) \cap E^{*}$.

Consider the problem

$$
\begin{aligned}
& \alpha^{\prime}(x)=\sigma(x, \alpha(x))+\varepsilon_{0}, \quad x \in J \backslash J_{\mathrm{imp}}, \\
& \alpha(0)=\varepsilon_{1}, \\
& \Delta \alpha(x)=\sigma_{0}\left(x, \alpha\left(x^{-}\right)\right)+\varepsilon_{2}, \quad x \in J_{\mathrm{imp}} .
\end{aligned}
$$

There exists $\widetilde{\varepsilon}>0$ such that for $0<\varepsilon_{i}<\widetilde{\varepsilon}, i=0,1$, 2, there exists a solution $\omega(\cdot ; \varepsilon), \varepsilon=\left(\varepsilon_{0}, \varepsilon_{1}, \varepsilon_{2}\right)$, of $(16)$ and this solution is defined on $\left[0, a_{0}\right)$. 
Let

$$
\widetilde{v}(x, y)= \begin{cases}v(x, y)+\varepsilon_{0}, & (x, y) \in E_{0}, \\ v(x, y)+\omega(x ; \varepsilon), & (x, y) \in\left(\left[0, a_{0}\right) \times \mathbb{R}^{n}\right) \cap\left(E \cup \partial_{0} E\right) .\end{cases}
$$

We prove that

$$
u(x, y)<\widetilde{v}(x, y) \quad \text { on }\left(\left[0, a_{0}\right) \times \mathbb{R}^{n}\right) \cap\left(E \cup \partial_{0} E\right) .
$$

We have

$$
\begin{aligned}
F[u](x, y)-F[\widetilde{v}](x, y) \\
=F[u](x, y)-D_{x} v(x, y)-\omega^{\prime}(x ; \varepsilon) \\
\quad+f\left(x, y, \widetilde{v}(x, y), \widetilde{v}_{(x, y)}, D_{y} v(x, y), D_{y y} v(x, y)\right) \\
\leq F[u](x, y)-D_{x} v(x, y)-\omega^{\prime}(x ; \varepsilon) \\
\quad+f\left(x, y, v(x, y), v_{(x, y)}, D_{y} v(x, y), D_{y y} v(x, y)\right)+\sigma(x, \omega(x ; \varepsilon)) \\
=F[u](x, y)-F[v](x, y)-\varepsilon_{0}<0, \quad(x, y) \in\left(E \backslash E_{\text {imp }}\right) \cap\left(\left[0, a_{0}\right) \times \mathbb{R}^{n}\right) .
\end{aligned}
$$

For $(x, y) \in E_{\text {imp }}$ we have

$$
\begin{aligned}
G[u](x, y)-G[\widetilde{v}](x, y) & \\
= & G[u](x, y)-\Delta \widetilde{v}(x, y)+g\left(x, y, \widetilde{v}\left(x^{-}, y\right), \widetilde{v}_{\left(x^{-}, y\right)}\right) \\
\leq & G[u](x, y)-\Delta v(x, y)-\Delta \omega(x ; \varepsilon)+g\left(x, y, v\left(x^{-}, y\right), v_{\left(x^{-}, y\right)}\right) \\
& \quad+\sigma_{0}\left(x, \omega\left(x^{-} ; \varepsilon\right)\right) \\
= & G[u](x, y)-G[v](x, y)-\varepsilon_{2}<0 .
\end{aligned}
$$

Since $u(x, y)<\widetilde{v}(x, y)$ on $\left(E_{0} \cup \partial_{0} E\right) \cap\left(\left[0, a_{0}\right) \times \mathbb{R}^{n}\right)$, from Theorem 1 we have assertion (17). Since $\lim _{\varepsilon \rightarrow 0} \omega(x ; \varepsilon)=0$ uniformly with respect to $x$ on $\left[0, a_{0}\right)$, we obtain $(15)$. The constant $a_{0} \in\left(x_{k}, a\right)$ is arbitrary and therefore the proof is complete.

Assumption H3. Suppose that:

1. $\widetilde{\sigma}:\left([0, a] \backslash J_{\mathrm{imp}}\right) \times \mathbb{R}_{-} \rightarrow \mathbb{R}_{+}, \mathbb{R}_{-}=(-\infty, 0]$, is continuous, $\widetilde{\sigma}(x, 0)=0$ for $x \in[0, a] \backslash J_{\text {imp }}$ and for $p \leq \bar{p}$ we have

$$
f(x, y, p, w, q, s)-f(x, y, \bar{p}, w, q, s) \leq \widetilde{\sigma}(x, p-\bar{p}) \quad \text { on } \Omega,
$$

2. $\tilde{\sigma}_{0}: J_{\mathrm{imp}} \times \mathbb{R}_{-} \rightarrow \mathbb{R}_{+}$is continuous, $\widetilde{\sigma}_{0}(x, 0)=0$ for $x \in J_{\mathrm{imp}}$ and for $p \leq \bar{p}$ we have

$$
g(x, y, p, w)-g(x, y, \bar{p}, w) \leq \widetilde{\sigma}_{0}(x, p-\bar{p}) \quad \text { on } E_{\mathrm{imp}} \times \mathbb{R} \times C_{\mathrm{imp}}^{*}\left[B^{(-)}, \mathbb{R}\right]
$$


3. the left-hand minimal solution of the problem

$$
\begin{aligned}
& \alpha^{\prime}(x)=\widetilde{\sigma}(x, \alpha(x)), \quad x \in J \backslash J_{\mathrm{imp}}, \\
& \Delta \alpha(x)=\widetilde{\sigma}_{0}\left(x, \alpha\left(x^{-}\right)\right), \quad x \in J_{\mathrm{imp}}, \\
& \lim _{x \rightarrow a^{-}} \alpha(x)=0
\end{aligned}
$$

is $\alpha(x)=0, x \in J$.

THEOREM 3. Suppose that:

1. Assumptions $\mathrm{H} 1$ and $\mathrm{H} 3$ hold,

2. $u, v \in C_{\mathrm{imp}}^{(1,2)}\left[E^{*}, \mathbb{R}\right]$ satisfy the initial-boundary inequality (6), and the functional differential inequality (13) holds on $E \backslash E_{\mathrm{imp}}$,

3. estimate (14) is satisfied,

4. $f$ is parabolic with respect to $v$ in $E \backslash E_{\mathrm{imp}}$. Then

$$
u(x, y)<v(x, y) \quad \text { on } E^{*} .
$$

Proof. First we prove (18) for $(x, y) \in\left([0, a-\varepsilon) \times \mathbb{R}^{n}\right) \cap E$, where $a-x_{k}>\varepsilon>0$. Let $0<p_{0}<\min \left\{v(x, y)-u(x, y):(x, y) \in E_{0} \cup \partial_{0} E\right\}$. For $\delta>0$ denote by $\omega(\cdot ; \delta)$ the right-hand minimal solution of the problem

$$
\begin{aligned}
& \alpha^{\prime}(x)=-\widetilde{\sigma}(x,-\alpha(x))-\delta, \quad x \in J \backslash J_{\mathrm{imp}}, \\
& \alpha(0)=p_{0}, \\
& \Delta \alpha(x)=-\widetilde{\sigma}_{0}\left(x,-\alpha\left(x^{-}\right)\right)-\delta, \quad x \in J_{\mathrm{imp}} .
\end{aligned}
$$

If $p_{0}>0$ is fixed then to every $\varepsilon>0$ corresponds $\delta_{0}>0$ such that for $0<$ $\delta<\delta_{0}$ the solution $\omega(\cdot ; \delta)$ of $(19)$ exists and is positive on $[0, a-\varepsilon)$. Suppose that $\delta>0$ is a constant such that $\omega(\cdot ; \delta)$ satisfies the above conditions. Let

$$
\widetilde{u}(x, y)= \begin{cases}u(x, y)+p_{0}, & (x, y) \in E_{0}, \\ u(x, y)+\omega(x ; \delta), & (x, y) \in\left(E \cup \partial_{0} E\right) \cap\left([0, a-\varepsilon) \times \mathbb{R}^{n}\right) .\end{cases}
$$

We will prove that

$$
\widetilde{u}(x, y)<v(x, y) \quad \text { on } E \cap\left([0, a-\varepsilon) \times \mathbb{R}^{n}\right) .
$$

It follows from $\mathrm{H} 1$ and $\mathrm{H} 3$ that

$$
\begin{aligned}
F[\widetilde{u}](x, y)-F[v](x, y) \leq & D_{x} u(x, y)+\omega^{\prime}(x ; \delta) \\
& -f\left(x, y, u(x, y), u_{(x, y)}, D_{y} u(x, y), D_{y y} u(x, y)\right) \\
& +\widetilde{\sigma}(x,-\omega(x ; \delta))-F[v](x, y) \\
= & F[u](x, y)-F[v](x, y)-\delta<0
\end{aligned}
$$

for $(x, y) \in\left(E \backslash E_{\text {imp }}\right) \cap\left([0, a-\varepsilon) \times \mathbb{R}^{n}\right)$.

Now we prove that

$$
G[\widetilde{u}](x, y)<G[v](x, y), \quad(x, y) \in E_{\mathrm{imp}} .
$$


It follows from H3, (14) and (19) that

$$
\begin{aligned}
G[\widetilde{u}](x, y)-G[v](x, y) \leq & \Delta u(x, y)+\Delta \omega(x ; \delta)-g\left(x, y, u\left(x^{-}, y\right), u_{\left(x^{-}, y\right)}\right) \\
& +\widetilde{\sigma}_{0}\left(x,-\omega\left(x^{-} ; \delta\right)\right)-G[v](x, y) \\
= & G[u](x, y)-G[v](x, y)-\delta<0, \quad(x, y) \in E_{\mathrm{imp}}
\end{aligned}
$$

which completes the proof of $(21)$. Since $\widetilde{u}(x, y)<v(x, y)$ for $(x, y) \in$ $\left(E_{0} \cup \partial_{0} E\right) \cap\left([0, a-\varepsilon) \times \mathbb{R}^{n}\right)$, we have estimate $(20)$ from Theorem 1 . It follows from $(20)$ that $u(x, y)<v(x, y)$ on $\left([0, a-\varepsilon) \times \mathbb{R}^{n}\right) \cap E$. Since $\varepsilon>0$ is arbitrary, inequality (18) holds on $E^{*}$.

3.2. Comparison theorems for parabolic functional differential inequalities. In this section we prove estimates of functions satisfying impulsive parabolic functional differential inequalities by means of solutions of impulsive ordinary functional differential equations.

Let $C_{\text {imp }}\left[J_{0} \cup J, \mathbb{R}\right]$ be the class of all functions $\alpha: J_{0} \cup J \rightarrow \mathbb{R}$ such that:

(i) the restriction of $\alpha$ to $J_{0} \cup J \backslash J_{\text {imp }}$ is continuous,

(ii) for each $x \in J_{\text {imp }}$ the limits

$$
\lim _{\substack{t \rightarrow x \\ t<x}} \alpha(t)=\alpha\left(x^{-}\right), \quad \lim _{\substack{t \rightarrow x \\ t>x}} \alpha(t)=\alpha\left(x^{+}\right)
$$

exist and $\alpha(x)=\alpha\left(x^{+}\right)$for $x \in J_{\text {imp }}$.

Suppose that we have a sequence $\left\{t_{1}, \ldots, t_{r}\right\}$ such that $-\tau_{0} \leq t_{1}<t_{2}<$ $\ldots<t_{r} \leq 0$. For $t_{1}>-\tau_{0}$ we also define $t_{0}=-\tau_{0}$ and for $t_{r}<0$ we put $t_{r+1}=0$. Let $J^{(i)}=\left(t_{i}, t_{i+1}\right), i=0,1, \ldots, r$.

We denote by $C_{\mathrm{imp}}^{*}\left[J_{0}, \mathbb{R}\right]$ the class of all functions $\eta: J_{0} \rightarrow \mathbb{R}$ such that there exists a sequence $\left\{t_{0}, t_{1}, \ldots, t_{r}, t_{r+1}\right\}$ depending on $\eta$ such that:

(i) the functions $\eta_{\mid J^{(i)}}, i=0,1, \ldots, r$, are continuous,

(ii) for each $i, i=2, \ldots, r+1$, and for $t_{1}>-\tau_{0}$, the limit

$$
\lim _{\substack{t \rightarrow t_{i} \\ t<t_{i}}} \eta(t)=\eta\left(t_{i}^{-}\right)
$$

exists,

(iii) for each $i, i=0,1, \ldots, r-1$, and for $t_{r}<0$, the limit

$$
\lim _{\substack{t \rightarrow t_{i} \\ t>t_{i}}} \eta(t)=\eta\left(t_{i}^{+}\right)
$$

exists and $\eta\left(t_{i}\right)=\eta\left(t_{i}^{+}\right)$.

For $\tau_{0}>0$ we put $J_{0}^{(-)}=\left[-\tau_{0}, 0\right)$ and $C_{\text {imp }}^{*}\left[J_{0}^{(-)}, \mathbb{R}\right]=\left\{\eta_{\mid J_{0}^{(-)}}: \eta \in\right.$ $\left.C_{\text {imp }}^{*}\left[J_{0}, \mathbb{R}\right]\right\}$. We will denote the elements of $C_{\text {imp }}^{*}\left[J_{0}, \mathbb{R}\right]$ and $C_{\text {imp }}^{*}\left[J_{0}^{(-)}, \mathbb{R}\right]$ by the same symbols. We denote by $\|\cdot\|_{0}$ the supremum norm in the space $C_{\mathrm{imp}}^{*}\left[J_{0}, \mathbb{R}\right]$ and in the space $C_{\mathrm{imp}}^{*}\left[J_{0}^{(-)}, \mathbb{R}\right]$. For $z \in C_{\mathrm{imp}}\left[E^{*}, \mathbb{R}\right]$ we define 
$T z: J_{0} \cup J \rightarrow \mathbb{R}_{+}$by

$$
(T z)(x)=\max \{|z(x, y)|: y \in[-c, c]\}, \quad x \in\left[-\tau_{0}, a\right) .
$$

If $\alpha: J_{0} \cup J \rightarrow \mathbb{R}$ and $x \in J$ then we define $\alpha_{(x)}: J_{0} \rightarrow \mathbb{R}$ by $\alpha_{(x)}(t)=$ $\alpha(x+t), t \in J_{0}$. For the above $\alpha$ and $x$ we define $\alpha_{\left(x^{-}\right)}: J_{0}^{(-)} \rightarrow \mathbb{R}$ by $\alpha_{\left(x^{-}\right)}(t)=\alpha(x+t)$ for $t \in J_{0}^{(-)}$. For $w \in C_{\mathrm{imp}}^{*}[B, \mathbb{R}]$ we define $T^{*} w: J_{0} \rightarrow$ $\mathbb{R}_{+}$by

$$
\left(T^{*} w\right)(t)=\max \{|w(t, s)|: s \in[-\tau, \tau]\} .
$$

Lemma 1. If $z \in C_{\mathrm{imp}}\left[E^{*}, \mathbb{R}\right]$ then $T z \in C_{\mathrm{imp}}\left[J_{0} \cup J, \mathbb{R}_{+}\right]$. If $w \in$ $C_{\mathrm{imp}}^{*}[B, \mathbb{R}]$ then $T^{*} w \in C_{\mathrm{imp}}^{*}\left[J_{0}, \mathbb{R}_{+}\right]$.

We omit the proof.

Assumption H4. Suppose that:

1. the functions $\sigma:\left([0, a) \backslash J_{\mathrm{imp}}\right) \times \mathbb{R}_{+} \times C_{\mathrm{imp}}^{*}\left[J_{0}, \mathbb{R}_{+}\right] \rightarrow \mathbb{R}_{+}$and $\widetilde{\sigma}: J_{\mathrm{imp}} \times \mathbb{R}_{+} \times C_{\mathrm{imp}}^{*}\left[J_{0}^{(-)}, \mathbb{R}_{+}\right] \rightarrow \mathbb{R}_{+}$are continuous and non-decreasing with respect to the functional argument,

2. for each $(x, \eta) \in J \times C_{\mathrm{imp}}^{*}\left[J_{0}^{(-)}, \mathbb{R}_{+}\right]$the function $\gamma(p)=p+\widetilde{\sigma}(x, p, \eta)$, $p \in \mathbb{R}_{+}$, is non-decreasing on $\mathbb{R}_{+}$.

Lemma 2. Suppose that:

1. Assumption $\mathrm{H} 4$ holds and $\psi \in C_{\mathrm{imp}}\left[J_{0} \cup J, \mathbb{R}\right]$,

2. $\widetilde{\eta} \in C\left(J_{0}, \mathbb{R}_{+}\right)$and $\omega(\cdot ; \widetilde{\eta}):\left[-\tau_{0}, a\right) \rightarrow \mathbb{R}_{+}$is the maximal solution of the problem

$$
\begin{aligned}
& \alpha^{\prime}(x)=\sigma\left(x, \alpha(x), \alpha_{(x)}\right), \quad x \in J \backslash J_{\mathrm{imp}}, \\
& \alpha(x)=\widetilde{\eta}(x), \quad x \in J_{0}, \\
& \Delta \alpha(x)=\widetilde{\sigma}\left(x, \alpha\left(x^{-}\right), \alpha_{\left(x^{-}\right)}\right), \quad x \in J_{\mathrm{imp}},
\end{aligned}
$$

3. the function $\psi$ satisfies

$$
\begin{gathered}
\psi(x) \leq \widetilde{\eta}(x), \quad x \in J_{0}, \\
\Delta \psi(x) \leq \widetilde{\sigma}\left(x, \psi\left(x^{-}\right), \psi_{\left(x^{-}\right)}\right), \quad x \in J_{\mathrm{imp}},
\end{gathered}
$$

4. for $x \in P_{+}=\left\{x>0, x \in J \backslash J_{\mathrm{imp}}: \psi(x)>\omega(x ; \widetilde{\eta})\right\}$ we have

$$
D_{-} \psi(x) \leq \sigma\left(x, \psi(x), \psi_{(x)}\right),
$$

where $D_{-}$is the left-hand lower Dini derivative.

Then $\psi(x) \leq \omega(x ; \widetilde{\eta})$ for $x \in\left[-\tau_{0}, a\right)$.

We omit the proof. 
THEOREM 4. Suppose that:

1. Assumption $\mathrm{H} 4$ holds, $f \in C(\Omega, \mathbb{R})$ and for each $(x, y, p, w) \in(E \backslash$ $\left.E_{\mathrm{imp}}\right) \times \mathbb{R} \times C_{\mathrm{imp}}^{*}[B, \mathbb{R}]$ we have

$$
f(x, y, p, w, 0,0) \operatorname{sign} p \leq \sigma\left(x,|p|, T^{*} w\right),
$$

where $\operatorname{sign} p$ denotes 1 if $p \geq 0$ and -1 if $p<0$,

2. $u \in C_{\mathrm{imp}}^{(1,2)}\left[E^{*}, \mathbb{R}\right]$ and

(24) $\quad D_{x} u(x, y)=f\left(x, y, u(x, y), u_{(x, y)}, D_{y} u(x, y), D_{y y} u(x, y)\right)$,

for $(x, y) \in E \backslash E_{\mathrm{imp}}$,

3. $\tilde{\eta} \in C\left(J_{0}, \mathbb{R}_{+}\right)$and

$$
|u(x, y)| \leq \widetilde{\eta}(x), \quad(x, y) \in E_{0},
$$

4. $\omega(\cdot ; \widetilde{\eta}):\left[-\tau_{0}, a\right) \rightarrow \mathbb{R}_{+}$is the maximal solution of the problem $(22)$,

5. the boundary estimate

$$
|u(x, y)| \leq \omega(x ; \widetilde{\eta}), \quad(x, y) \in \partial_{0} E
$$

and the impulsive estimate

(27) $|u(x, y)|$

$$
\leq\left|u\left(x^{-}, y\right)\right|+\widetilde{\sigma}\left(x,\left|u\left(x^{-}, y\right)\right|,\left(T^{*} u\right)_{\left(x^{-}\right)}\right), \quad(x, y) \in E_{\mathrm{imp}} \cup \partial_{0} E_{\mathrm{imp}},
$$

are satisfied,

6. $f$ is parabolic with respect to $u$ in $E \backslash E_{\mathrm{imp}}$.

Then

$$
|u(x, y)| \leq \omega(x ; \widetilde{\eta}) \quad \text { for }(x, y) \in E^{*} .
$$

Proof. We prove that the function $\psi=T u$ satisfies all conditions of Lemma 2. It follows from (25) and (27) that condition 3 of Lemma 2 holds. Suppose that $x \in P_{+}$. There exists $y \in[-c, c]$ such that $\psi(x)=|u(x, y)|$. It follows from $(26)$ that $y \in(-b, b)$. There are two possibilities: either

$$
\psi(x)=u(x, y)
$$

or

$$
\psi(x)=-u(x, y) .
$$

Suppose that $(29 \mathrm{~b})$ holds. Then $D_{y} u(x, y)=0$,

$$
\sum_{i, j=1}^{n} D_{y_{i} y_{j}} u(x, y) \lambda_{i} \lambda_{j} \geq 0, \quad \lambda=\left(\lambda_{1}, \ldots \lambda_{n}\right) \in \mathbb{R}^{n}
$$

and

$$
\begin{aligned}
D_{-} \psi(x) & \leq-D_{x} u(x, y) \\
& =-f\left(x, y, u(x, y), u_{(x, y)}, D_{y} u(x, y), D_{y y} u(x, y)\right)
\end{aligned}
$$




$$
\begin{aligned}
& \leq-f\left(x, y, u(x, y), u_{(x, y)}, 0,0\right) \\
& =f\left(x, y, u(x, y), u_{(x, y)}, 0,0\right) \operatorname{sign} u(x, y) \\
& \leq \sigma\left(x,|u(x, y)|,(T u)_{(x)}\right) .
\end{aligned}
$$

Thus $\psi$ satisfies condition 4 of Lemma 2. The case when (29a) holds is analogous. Thus all conditions of Lemma 2 are satisfied and (28) follows.

Let us consider two problems: the problem (3)-(5) and the problem

(30) $D_{x} z(x, y)=\widetilde{f}\left(x, y, z(x, y), z_{(x, y)}, D_{y} z(x, y), D_{y y} z(x, y)\right)$,

$$
(x, y) \in E \backslash E_{\mathrm{imp}},
$$

(31) $z(x, y)=\widetilde{\varphi}(x, y), \quad(x, y) \in E_{0} \cup \partial_{0} E$,

(32) $\Delta z(x, y)=\widetilde{g}\left(x, y, z\left(x^{-}, y\right), z_{\left(x^{-}, y\right)}\right), \quad(x, y) \in E_{\mathrm{imp}} \cup \partial_{0} E_{\mathrm{imp}}$,

where $\tilde{f}: \Omega \rightarrow \mathbb{R}$ and $\widetilde{g}: \Omega_{\mathrm{imp}} \rightarrow \mathbb{R}$ and $\widetilde{\varphi}: E_{0} \cup \partial_{0} E \rightarrow \mathbb{R}$, where $\widetilde{\varphi}_{\mid \partial_{0} E} \in C_{\mathrm{imp}}\left[\partial_{0} E, \mathbb{R}\right]$, are given functions.

We prove an estimate of the difference between solutions of (3)-(5) and (30)-(32).

THEOREM 5. Suppose that:

1. Assumption $\mathrm{H} 4$ holds,

2. $f, \widetilde{f} \in C(\Omega, \mathbb{R}), g, \widetilde{g} \in C\left(\Omega_{\mathrm{imp}}, \mathbb{R}\right)$ satisfy the inequalities

$$
\begin{array}{r}
(f(x, y, p, w, q, s)-\widetilde{f}(x, y, \bar{p}, \bar{w}, q, s)) \operatorname{sign}(p-\bar{p}) \\
\quad \leq \sigma\left(x,|p-\bar{p}|, T^{*}(w-\bar{w})\right) \quad \text { on } \Omega, \\
|g(x, y, p, w)-\widetilde{g}(x, y, \bar{p}, \bar{w})| \leq \widetilde{\sigma}\left(x,|p-\bar{p}|, T^{*}(w-\bar{w})\right) \quad \text { on } \Omega_{\mathrm{imp}},
\end{array}
$$

3. $\varphi, \widetilde{\varphi}: E_{0} \cup \partial_{0} E \rightarrow \mathbb{R}, \widetilde{\eta} \in C\left(J_{0}, \mathbb{R}_{+}\right), \varphi_{\mid E_{0}}, \widetilde{\varphi}_{\mid E_{0}} \in C\left(E_{0}, \mathbb{R}\right), \varphi_{\mid \partial_{0} E}$, $\widetilde{\varphi}_{\mid \partial_{0} E} \in C_{\mathrm{imp}}\left[\partial_{0} E, \mathbb{R}\right]$, and

$$
|\varphi(x, y)-\widetilde{\varphi}(x, y)| \leq \widetilde{\eta}(x), \quad(x, y) \in E_{0},
$$

4. the maximal solution $\omega(\cdot ; \widetilde{\eta})$ of $(22)$ is defined on $\left[-\tau_{0}, a\right)$ and $u, \widetilde{u} \in C_{\mathrm{imp}}^{(1,2)}\left[E^{*}, \mathbb{R}\right]$ are solutions of (3)-(5) and (28)-(30), respectively,

5. $|\varphi(x, y)-\widetilde{\varphi}(x, y)| \leq \omega(x ; \widetilde{\eta})$ on $\partial_{0} E$,

6. $f$ is parabolic with respect to $u$ in $E \backslash E_{\mathrm{imp}}$.

Then $|u(x, y)-\widetilde{u}(x, y)| \leq \omega(x ; \widetilde{\eta})$ for $(x, y) \in E^{*}$.

Proof. We prove that the function $\psi=T(u-\widetilde{u})$ satisfies all conditions of Lemma 2. It is easy to see that condition 3 of Lemma 2 holds. Suppose that $x \in P_{+}$. There exists $y \in[-c, c]$ such that $\psi(x)=|u(x, y)-\widetilde{u}(x, y)|$. From condition 5 of the theorem it follows that $y \in(-b, b)$. There are two possibilities: either

$$
\psi(x)=u(x, y)-\widetilde{u}(x, y)
$$


or

$$
\psi(x)=-(u(x, y)-\widetilde{u}(x, y)) .
$$

Suppose that $(34 \mathrm{a})$ holds. Then $D_{y}(u-\widetilde{u})(x, y)=0$,

$$
\sum_{i, j=1}^{n} D_{y_{i} y_{j}}(u-\widetilde{u})(x, y) \lambda_{i} \lambda_{j} \leq 0, \quad \lambda=\left(\lambda_{1}, \ldots, \lambda_{n}\right) \in \mathbb{R}^{n},
$$

and

$$
\begin{aligned}
D_{-} \psi(x) \leq & D_{x} u(x, y)-D_{x} \widetilde{u}(x, y) \\
= & f\left(x, y, u(x, y), u_{(x, y)}, D_{y} u(x, y), D_{y y} u(x, y)\right) \\
& \left.-f\left(x, y, u(x, y), u_{(x, y)}, D_{y} u(x, y), D_{y y} \widetilde{u}(x, y)\right)\right] \\
& +\left[f\left(x, y, u(x, y), u_{(x, y)}, D_{y} u(x, y), D_{y y} \widetilde{u}(x, y)\right)\right. \\
& \left.-\widetilde{f}\left(x, y, \widetilde{u}(x, y), \widetilde{u}_{(x, y)}, D_{y} u(x, y), D_{y y} \widetilde{u}(x, y)\right)\right] .
\end{aligned}
$$

The first difference in brackets is non-positive by the parabolicity of $f$ with respect to $u$. Since $u(x, y) \geq \widetilde{u}(x, y)$ by $(34 \mathrm{a})$, in view of condition 2 we get

$$
D_{-} \psi(x) \leq \sigma\left(x, \psi(x),(T \psi)_{(x)}\right), \quad x \in P_{+} .
$$

The case when (34b) holds is analogous. Thus all conditions of Lemma 2 are satisfied and the statement of the theorem follows.

THEOREM 6. Suppose that:

1. Assumption $\mathrm{H} 4$ holds,

2. $f \in C(\Omega, \mathbb{R}), g \in C\left(\Omega_{\mathrm{imp}}, \mathbb{R}\right)$ and

$$
\begin{aligned}
(f(x, y, p, w, q, s)-f(x, y, \bar{p}, \bar{w}, q, s)) \operatorname{sign}(p-\bar{p}) & \\
& \leq \sigma\left(x,|p-\bar{p}|, T^{*}(w-\bar{w})\right) \quad \text { on } \Omega, \\
|g(x, y, p, w)-g(x, y, \bar{p}, \bar{w})| & \leq \widetilde{\sigma}\left(x,|p-\bar{p}|, T^{*}(w-\bar{w})\right) \quad \text { on } \Omega_{\mathrm{imp}},
\end{aligned}
$$

3. $\sigma(x, 0, \theta)=0$ for $x \in J \backslash J_{\mathrm{imp}}$ and $\widetilde{\sigma}(x, 0, \theta)=0$ for $x \in J_{\mathrm{imp}}$, where $\theta(t)=0$ for $t \in J_{0}$,

4. the maximal solution of the problem

$$
\begin{aligned}
& \alpha^{\prime}(x)=\sigma\left(x, \alpha(x), \alpha_{(x)}\right), \quad x \in J \backslash J_{\mathrm{imp}}, \\
& \alpha(x)=0, \quad x \in J_{0}, \\
& \Delta \alpha(x)=\tilde{\sigma}\left(x, \alpha\left(x^{-}\right), \alpha_{\left(x^{-}\right)}\right), \quad x \in J_{\mathrm{imp}},
\end{aligned}
$$

is $\alpha(x)=0, x \in J_{0} \cup J$.

Then the problem $(3)-(5)$ admits at most one solution in $C_{\mathrm{imp}}^{(1,2)}\left[E^{*}, \mathbb{R}\right]$.

Proof. Put $\tilde{f}=f$ and $\widetilde{g}=g$ and apply Theorem 5 .

Remark 2. Suppose that $\varrho:\left([0, a) \backslash J_{\text {imp }}\right) \times \mathbb{R}_{+} \times \mathbb{R}_{+} \rightarrow \mathbb{R}_{+}$and $\widetilde{\varrho}: J_{\mathrm{imp}} \times \mathbb{R}_{+} \times \mathbb{R}_{+} \rightarrow \mathbb{R}_{+}$are given functions and $\sigma:\left([0, a) \backslash J_{\mathrm{imp}}\right) \times \mathbb{R}_{+} \times$ 
$C_{\mathrm{imp}}^{*}\left[J_{0}, \mathbb{R}_{+}\right] \rightarrow \mathbb{R}_{+}$and $\tilde{\sigma}: J_{\mathrm{imp}} \times \mathbb{R}_{+} \times C_{\mathrm{imp}}^{*}\left[J_{0}^{(-)}, \mathbb{R}_{+}\right] \rightarrow \mathbb{R}_{+}$are defined by

$$
\begin{aligned}
& \sigma(x, p, \eta)=\varrho\left(x, p, \sup \left\{\eta(t): t \in J_{0}\right\}\right), \\
& \widetilde{\sigma}(x, p, \eta)=\widetilde{\varrho}\left(x, p, \sup \left\{\eta(t): t \in J_{0}^{(-)}\right\}\right) .
\end{aligned}
$$

Then:

1. Inequality (27) is equivalent to

$|u(x, y)| \leq\left|u\left(x^{-}, y\right)\right|+\widetilde{\varrho}\left(x,\left|u\left(x^{-}, y\right)\right|,\left\|u_{\left(x^{-}, y\right)}\right\|_{0}\right), \quad(x, y) \in E_{\mathrm{imp}} \cup \partial_{0} E_{\mathrm{imp}}$.

2. Estimates (23) and (33) are equivalent to

$$
f(x, y, p, w, 0,0) \operatorname{sign} p \leq \varrho\left(x,|p|,\|w\|_{0}\right),
$$

$(f(x, y, p, w, q, s)-\widetilde{f}(x, y, \bar{p}, \bar{w}, q, s)) \operatorname{sign}(p-\bar{p}) \leq \varrho\left(x,|p-\bar{p}|,\|w-\bar{w}\|_{0}\right)$, $|g(x, y, p, w)-\widetilde{g}(x, y, \bar{p}, \bar{w})| \leq \widetilde{\varrho}\left(x,|p-\bar{p}|,\|w-\bar{w}\|_{0}\right)$.

3. If we assume that $\widetilde{\eta} \in C\left(J_{0}, \mathbb{R}_{+}\right)$is non-decreasing on $J_{0}$ then the problem $(22)$ is equivalent to

$$
\begin{aligned}
& \alpha^{\prime}(x)=\varrho(x, \alpha(x), \alpha(x)), \quad x \in J \backslash J_{\mathrm{imp}}, \\
& \alpha(0)=\widetilde{\eta}(0), \\
& \Delta \alpha(x)=\widetilde{\varrho}\left(x, \alpha\left(x^{-}\right), \alpha\left(x^{-}\right)\right), \quad x \in J_{\mathrm{imp}} .
\end{aligned}
$$

Acknowledgements. The present investigation was partially supported by the Bulgarian Ministry of Education, Science and Technologies under Grant MM-422.

Z. Kamont wishes to express his thanks to the University of Gdańsk for the support of this research.

\section{References}

[1] D. Bainov, Z. Kamont and E. Minchev, On first order impulsive partial differential inequalities, Appl. Math. Comp. 61 (1994), 207-230.

[2] - - - - On impulsive parabolic differential inequalities, to appear.

[3] D. Bainov, V. Lakshmikantham and P. Simeonov, Theory of Impulsive Differential Equations, World Scientific, Singapore, 1989.

[4] D. Bainov and P. Simeonov, Systems with Impulse Effect: Stability, Theory and Applications, Ellis Horwood, Chichester, 1989.

[5] L. Byszewski, Impulsive degenerate nonlinear parabolic functional-differential inequalities, J. Math. Anal. Appl. 164 (1992), 549-559.

[6] —, System of impulsive nonlinear parabolic functional-differential inequalities, Comment. Math., to appear.

[7] C. Y. Chan and L. Ke, Remarks on impulsive quenching problems, in: First International Conference on Dynamic Systems and Applications, 1993, Atlanta, USA, to appear. 
[8] L. Erbe, H. Freedman, X. Liu and J. Wu, Comparison principles for impulsive parabolic equations with applications to models of single species growth, J. Austral. Math. Soc. Ser. B 32 (1991), 382-400.

[9] V. Lakshmikantham and S. Leela, Differential and Integral Inequalities, Vols. 1 and 2, Academic Press, New York, 1969.

[10] V. Mil'man and A. Myshkis, On the stability of motion in the presence of impulses, Sibirsk. Mat. Zh. 1 (2) (1960), 233-237 (in Russian).

[11] J. Szarski, Differential Inequalities, Polish Scientific Publishers, Warszawa, 1965.

D. D. Bainov and E. Minchev

Higher Medical Institute

P.O. Box 45

Sofia 1504, Bulgaria
Zdzisław Kamont Institute of Mathematics University of Gdańsk Wita Stwosza 57 80-952 Gdańsk, Poland 